\title{
Short-Term Load Forecasting Using Radial Basis Function Neural Network
}

\author{
Wen-Yeau Chang \\ Department of Electrical Engineering, St. John's University, Taiwan \\ Email:changwy@mail.sju.edu.tw
}

Received August 2015

\begin{abstract}
An accurate short-term forecasting method for load of electric power system can help the electric power system's operator to reduce the risk of unreliability of electricity supply. This paper proposed a radial basis function (RBF) neural network method to forecast the short-term load of electric power system. To demonstrate the effectiveness of the proposed method, the method is tested on the practical load data information of the Tai power system. The good agreements between the realistic values and forecasting values are obtained; the numerical results show that the proposed forecasting method is accurate and reliable.
\end{abstract}

\section{Keywords}

\section{Short-Term Load Forecasting, RBF Neural Network, Tai Power System}

\section{Introduction}

Electric power system load forecasting plays an important role in the electric energy management system, which has great influence on the operation, controlling and planning of electric power system [1]. Unexpected variations of the load may increase operating costs for the electricity system because the increased operation and maintain costs associated with cycling existing generation. Load forecasting is important in the allocation of balancing power. Besides, load forecasting is used for the hour-ahead scheduling of conventional power plants and trading of electricity on the spot market.

Recently, several methods have been employed for the load forecasting. The load forecasting methods can be generally categorized into two groups, time series based methods and artificial intelligence methods. Time series based methods include the auto regressive (AR) [2], auto regressive moving average (ARMA) [3], and auto regressive integrated moving average (ARIMA) [4]. With the development of artificial intelligence, various artificial intelligence methods for load prediction have been developed. The new developed methods include artificial neural network [1], fuzzy logic methods [5], least square support vector machine [6], and grey dynamic model [7]. The artificial intelligence methods are self-designing ones that can be automatically adjusted in changing system. To increase the accuracy of short-term load forecasting this paper takes the RBF Neural Network based method into account for the nonlinearity and periodicity in the load time series. 


\section{RBF Neural Network Based Load Forecasting Method}

RBF neural network is useful methodology for time series data forecasting. RBF neural network can be used to analysis the relationships between a major sequence and the other comparative sequences in a given set. Compared with back propagation neural networks, the RBF neural networks not only have faster training velocity and better approximation properties, but also can solve the local minima problem [8].

The RBF neural network is a forward network model with good performance, global approximation, and is free from the local minima problems. It is a multi-input, multi-output system consisting of an input layer, a hidden layer, and an output layer. During the data processing, the hidden layer performs nonlinear transforms for the feature extraction and the output layer gives a linear combination of output weights.

This paper uses the RBF neural network to forecast the load of electric power system. The architecture of a typical RBF neural network is shown in Figure 1 [9]. The network actually performs a nonlinear mapping from the input space $R^{d}$ to the output space $R^{m}$.

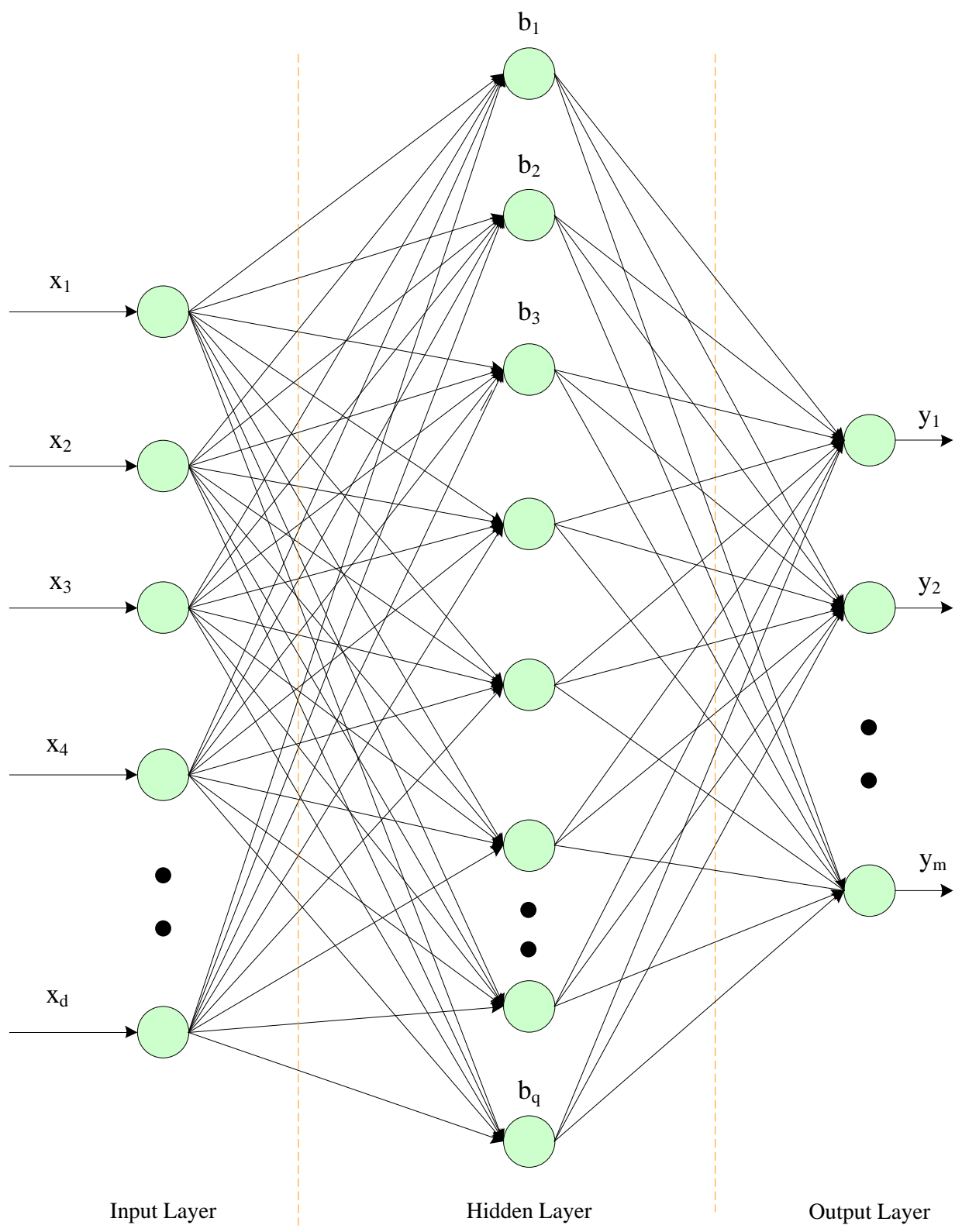

Figure 1. Architecture of the RBF neural network. 
In RBF neural network, each hidden neuron computes a Gaussian function in the following equation:

$$
b_{j}(\bar{x})=\exp \left[\frac{-\left(\bar{x}-\bar{\mu}_{j}\right)^{2}}{2 \sigma_{j}^{2}}\right], \text { for } \quad j=1,2, \cdots, q
$$

where $\mu_{j}$ and $\sigma_{j}$ are, respectively, the center and the width of the Gaussian potential function of the $j$ th neuron in the hidden layer.

Each output neuron of RBF neural network computes a linear function in the following form:

$$
o_{k}=\sum_{j=1}^{q} w_{k j} b_{j}(\bar{x})-\theta_{k}, \text { for } \quad k=1,2, \cdots, m
$$

where $o_{k}$ is output of the $k$ th node in the output layer, $w_{k j}$ is weight between $j$ th node in the hidden layer and $k$ th node in the output layer, $b_{j}(\bar{x})$ is output from the $j$ th node in the hidden layer, $\theta_{k}$ is bias of the $k$ th node in the output layer.

The RBF neural network based forecasting method has been successfully implemented for load forecasting. The RBF neural network models were developed for $10 \mathrm{~min}$. ahead load forecasting. The architecture of the RBF neural network-based load forecasting method is shown in Figure 2. The input layer has 4 neurons for the load of 20 minutes ago, 10 minutes ago, the current load and the temperature forecasting value; the hidden layer has 24 neurons and the output layer has one neuron for the 10minutes ahead load forecasting.

The RBF neural network based forecasting method is briefly described in the following steps:

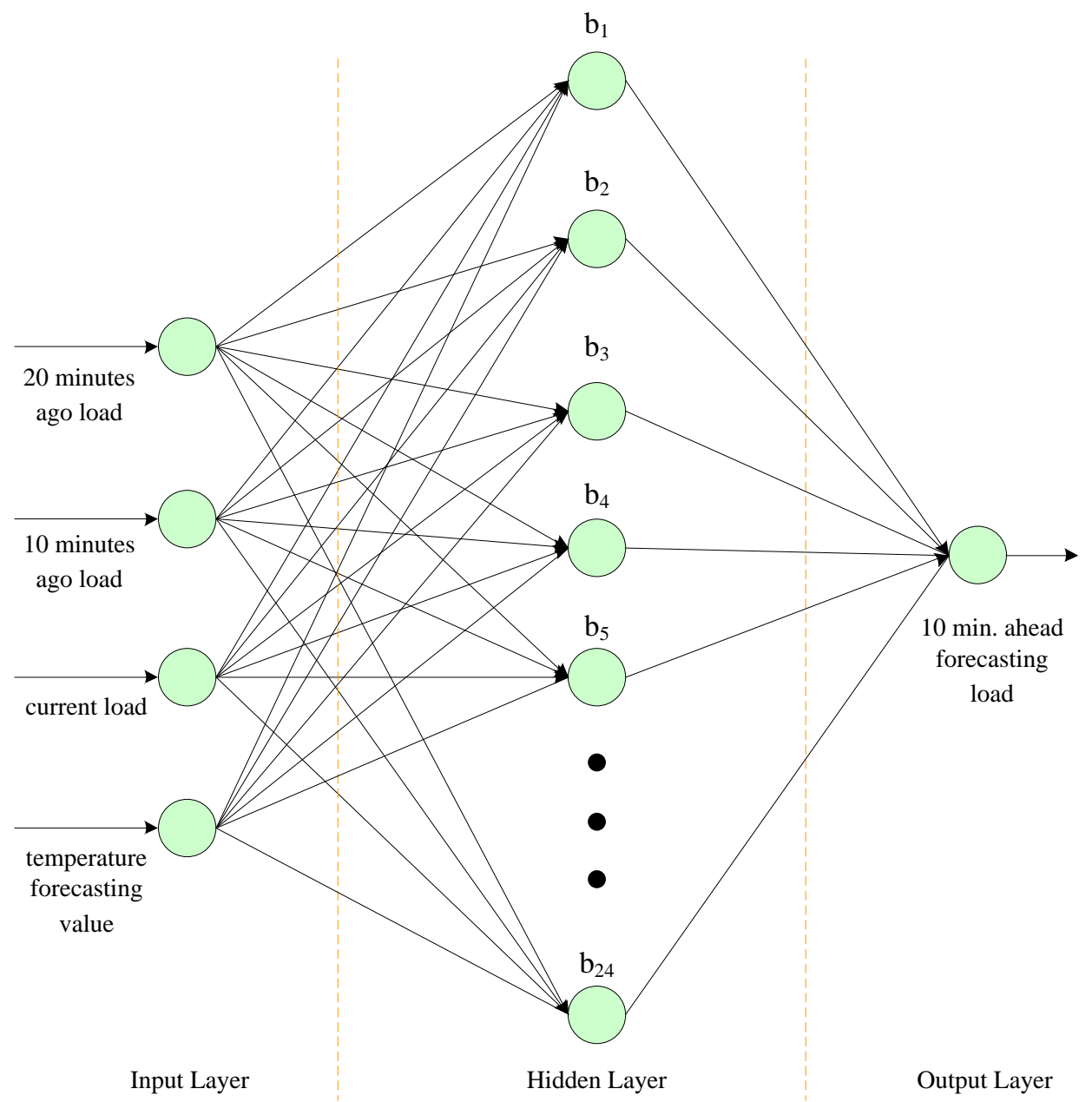

Figure 2. Architecture of the RBF neural network based load forecasting method. 
Step 1 Create a database of load data and temperature data of electric power system.

Step 2 Normalize all the load data and temperature data.

Step 3 Prepare the training set for the RBF neural network.

Step 4 Using the enhanced particle swarm optimization (EPSO) to train the RBF neural network for load forecasting.

Step 5 Save the Gaussian functions centers, widths and connection weights between the hidden and output layers of the trained RBF neural network, as the EPSO training procedure is finished.

Step 6 Use the trained RBF neural network to forecast the load of electric power system.

\section{Numerical Results}

To verify the proposed forecasting method, the method has been applied for load forecasting in Taiwan. The load forecasting is computed using the historical load data every $10 \mathrm{~min}$. of the electric power system in Taiwan-Tai power. The load time series data of Tai power system are recorded every $10 \mathrm{~min}$. For the sake of clear comparison, no exogenous variables are considered. Due to the area characteristic, the load data are divided into 4 categories: northern area, middle area, southern area, and full area. The weekday testing of four areas results are shown below.

In weekday testing, the following days are selected: May 11-15, 2015, corresponding to the typical weekday. The historical data set with 864 patterns are divided into training data set for RBF neural network composed of 720 patterns collected from May 11-14, and testing data set composed of 144 patterns collected from May15. The number of neurons in hidden layer of RBF neural network is 24 . The performance of the proposed method is evaluated using two indices, namely the maximum absolute percentage error and the mean absolute percentage error. Numerical results for northern area of Tai power system with the RBF neural network based method are shown in Figure 3. In this case the maximum absolute percentage error is $2.624 \%$ and the mean absolute percentage error is $1.007 \%$. Numerical results for middle area of Tai power system with the RBF neural network based method are shown in Figure 4. In this case the maximum absolute percentage error is $2.433 \%$ and the mean absolute percentage error is $1.075 \%$. Numerical results for southern area of Tai power system with the RBF neural network based method are shown in Figure 5. In this case the maximum absolute percentage error is $2.502 \%$ and the mean absolute percentage error is $0.955 \%$. Numerical results for full area of Tai power system with the RBF neural network based method are shown in Figure 6. In this case the maximum absolute percentage error is $2.575 \%$ and the mean absolute percentage error is $1.146 \%$. The Comparing the four area data, a general conclusion that may be drawn from the obtained results is that proposed forecasting method can forecast the load accurately.

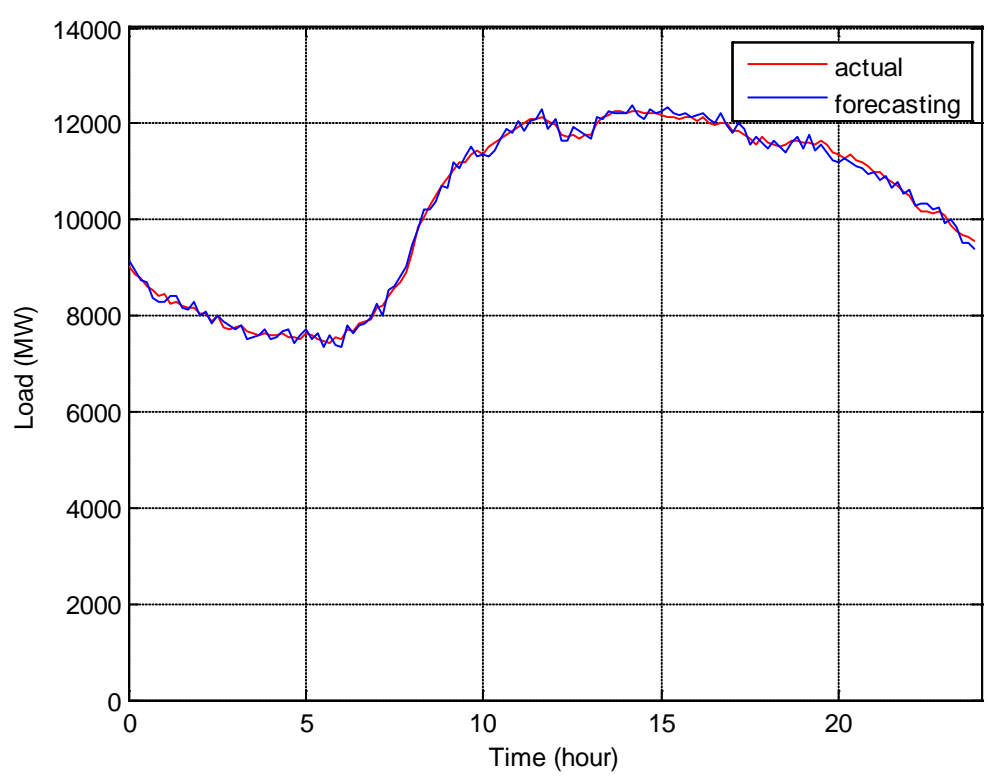

Figure 3. Load forecasting results for northern area of Tai power system. 


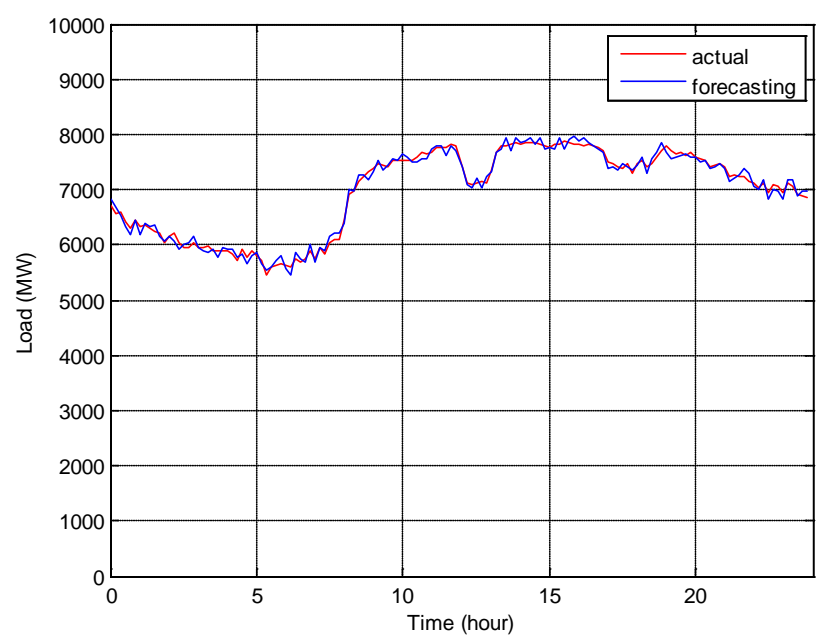

Figure 4. Load forecasting results for middle area of Tai power system.

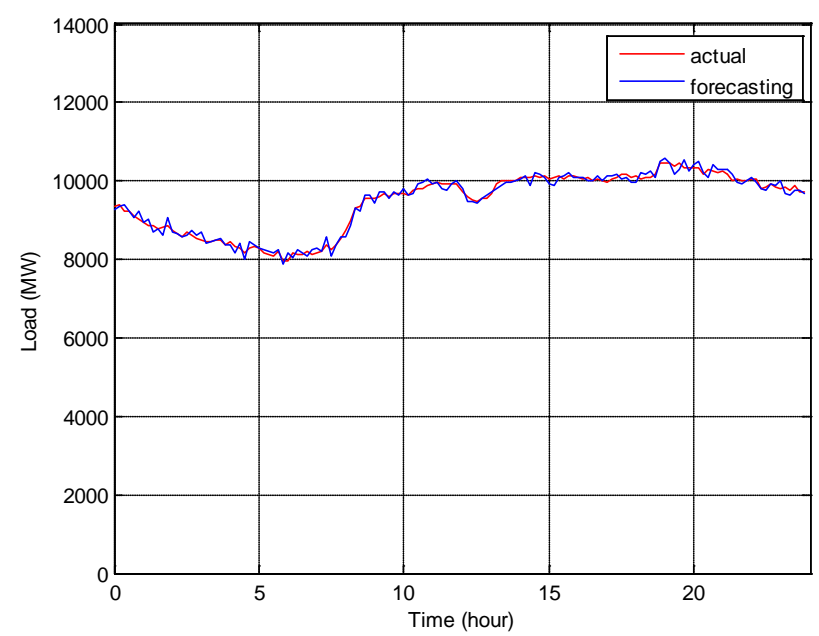

Figure 5. Load forecasting results for southern area of Tai power system.

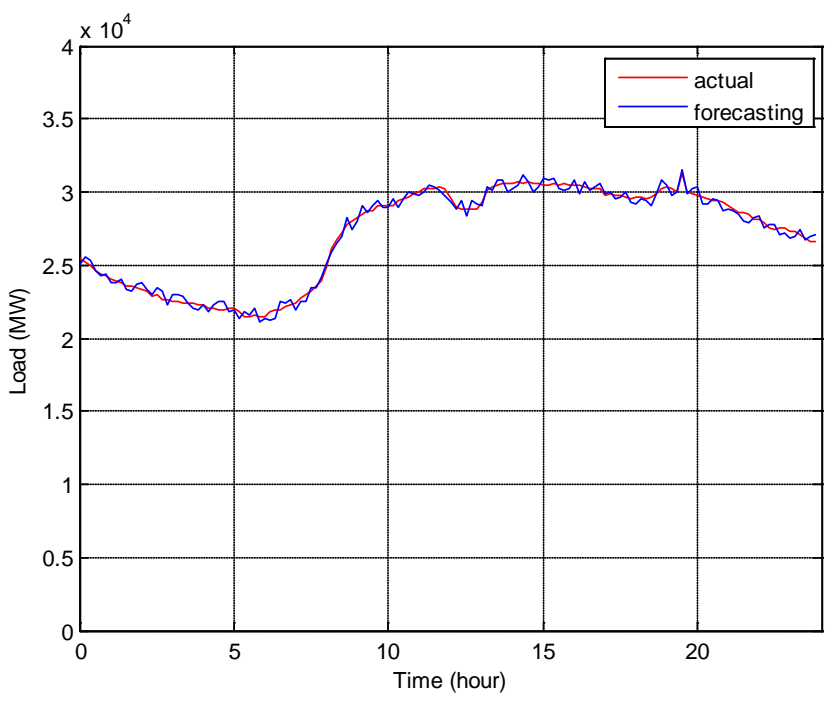

Figure 6. Load forecasting results for full area of Tai power system. 


\section{Conclusion}

Based on the RBF neural network scheme, this paper has proposed a method to accurately and reliably forecast the load of an electric power system. To verify the effectiveness of the proposed technique, the historical load data of the Tai power system is used, the RBF neural network based load forecasting method can forecast the load accurately and reliably. An evaluation of the forecast methods is performed, using practical load information for Tai power system. The results demonstrate the effectiveness of the proposed method and this method provided improved accuracy in the short-term load forecasting.

\section{Acknowledgements}

The author would like to express his acknowledgements to the Ministry of Science and Technology of Taiwan for the financial support under Grant MOST 103-2632-E-129-002-MY3.

\section{References}

[1] Dai, W. and Wang, P. (2007) Application of Pattern Recognition and Artificial Neural Network to Load Forecasting in Electric Power System. Proceedings of the Third International Conference on Natural Computation, Haikou, 24-27 August 2007, 381-385. http://dx.doi.org/10.1109/ICNC.2007.260

[2] Yu, J., Lee, H., Jeong, Y. and Kim, S.(2014) Short-Term Hourly Load Forecasting Using PSO-Based AR Model. Proceedings of the 15th International Symposium on Soft Computing and Intelligent Systems, Kitakyushu, 3-6 December 2014, 1449-1453. http://dx.doi.org/10.1109/scis-isis.2014.7044868

[3] Hafen, R.P., Samaan, N., Makarov, Y.V., Diao, R. and Lu, N. (2014) Joint Seasonal ARMA Approach for Modeling of Load Forecast Errors in Planning Studies. Proceedings of the 2014 IEEE PES T\&D Conference and Exposition, Chicago, 14-17 April 2014, 1-5. http://dx.doi.org/10.1109/tdc.2014.6863150

[4] Zhu, X. and Shen, M. (2012) Based on the ARIMA Model with Grey Theory for Short Term Load Forecasting Model. Proceedings of the 2012 International Conference on Systems and Informatics, Yantai, 19-20 May 2012, 564-567. http://dx.doi.org/10.1109/icsai.2012.6223060

[5] Khosravi, A., Nahavandi, S., Creighton, D. and Srinivasan, D. (2012) Interval Type-2 Fuzzy Logic Systems for Load Forecasting: A Comparative Study. IEEE Transactions on Power Systems, 27, 1274-1282. http://dx.doi.org/10.1109/TPWRS.2011.2181981

[6] Gao, R., Zhang, L. and Liu, X. (2012) Short-Term Load Forecasting Based on Least Square Support Vector Machine Combined with Fuzzy Control. Proceedings of the 2012 10th World Congress on Intelligent Control and Automation, Beijing, 6-8 July 2012, 1048-1051. http://dx.doi.org/10.1109/WCICA.2012.6358034

[7] Sabri, Y., Hariyanto, N. and Fitriana, F. (2011) Spatial Short-Term Load Forecasting Using Grey Dynamic Model Specific in Tropical Area. Proceedings of the 2011 International Conference on Electrical Engineering and Informatics, Bandung, 17-19 July 2011, 1-6. http://dx.doi.org/10.1109/ICEEI.2011.6021776

[8] Chang, W.Y. (2013) Estimation of the State of Charge for a LFP Battery Using a Hybrid Method that Combines a RBF Neural Network, an OLS Algorithm and AGA. International Journal of Electrical Power and Energy Systems, 53, 603-611. http://dx.doi.org/10.1016/j.ijepes.2013.05.038

[9] Chang, W.Y. (2013) Wind Energy Conversion System Power Forecasting Using Radial Basis Function Neural Network. Applied Mechanics and Materials, 284-287, 1067-1071. http://dx.doi.org/10.4028/www.scientific.net/AMM.284-287.1067 